\title{
Inhomogeneous Lacunary Interpolation and Optimization Errors Bound of Seventh Spline
}

\author{
Faraidun K. Hamasalh ${ }^{1, *}$, Karwan H.F. Jwamer ${ }^{2}$ \\ ${ }^{1}$ University of Sulaimani, Faculty of Science and Science Education, School of Science Education, Sulaimani, Iraq \\ ${ }^{2}$ University of Sulaimani, Faculty of Science and Science Education, School of Science, Sulaimani, Iraq \\ *Corresponding author: faraidunsalh@gmail.com
}

Received March 29, 2013; Revised June 09, 2013; Accepted June 11, 2013

\begin{abstract}
This paper surveys and reviews paper of spline degree seven inhomogeneous and optimized the best errors bound by spline $(0,2,5 ; 0,3,6)$ case. It has been shown that the existence, uniqueness and convergence analysis with minimizing the error bounds of deficient seventh spline interpolated.
\end{abstract}

Keywords: interpolation spline function, boundary condition, optimal error bounds

\section{Introduction}

Interpolation polynomial occurs naturally in many fields of physics and mathematical statistics. They also arise as representation formulas for the interpolating of data.

This theory has developed into an interesting branch of applicable mathematics to minimize the function, which contains a wealth of new idea for inspiration inhomogeneous lacunary interpolation by higher order spline function. A better accuracy in the interpolation is especially relevant since the spline function is fully expressed in terms of boundary quantities. This type of problem arises in the mathematical modeling of inhomogeneous lacunary interpolations concerning $[1,4,10,11]$. Spline function have been used for this purpose in minimize errors estimation [3,5,6]. Various types of splines, such as quadratic [2], quinitics [8],sixth [7] and ninth [9] have been used to interpolate the polynomial and solve these different kinds of problems. In [4] used six degree spline function for the $(0,2 ; 0,1,4)$ inhomogeneous lacunarcunary case but in the present paper we use seven degree spline for the $(0,2,5 ; 0,3,6)$ inhomogeneous lacunary type that means our model are differences as follows:

Form the Model, form the boundary conditions, form the Polynomials which we obtained, and all results from the Theorems in the next sections.

\section{Splines Theory}

In these extended set of polynomials, we found new polynomial with better approximation theoretic performances as seventh splines.

$$
\begin{aligned}
& S\left(\frac{v}{n}\right)=f_{v}, v=0,1, \ldots, n, \\
& S^{\prime \prime}\left(\frac{2 v}{n}\right)=f_{2 v}^{\prime \prime \prime}, S^{\prime \prime \prime}\left(\frac{2 v+1}{n}\right)=f_{2 v+1}^{\prime \prime \prime}, \\
& S^{(5)}\left(\frac{2 v}{n}\right)=f_{2 v}^{(5)}, S^{(6)}\left(\frac{2 v+1}{n}\right)=f_{2 v+1}^{(6)}
\end{aligned}
$$

where $v=0,1,2, \ldots .,\left(\frac{n-1}{2}\right), S^{\prime}(0)=f_{0}^{\prime}, S^{\prime}(1)=f_{n}^{\prime}$

We may all it $(0,2,5 ; 0,3,6)$ interpolation, in the next communication we shall return to same other problems of this nature: It can be verified that if $P(x)$ is seventh on [0, 1] then

$$
\begin{aligned}
P(x)= & P(0) B_{o}(x)+P(1) B_{1}(x) \\
& +P^{\prime \prime}(0) B_{2}(x)+P^{\prime \prime \prime}(1) B_{3}(x) \\
& +P^{(4)}(0) B_{4}(x)+P^{(4)}(1) B_{5}(x) \\
& +P^{(5)}(0) B_{6}(x)+P^{(6)}(1) B_{7}(x)
\end{aligned}
$$

Where

$$
\begin{aligned}
& B_{0}(x)=1-x, \\
& B_{1}(x)=x, \\
& B_{2}(x)=\frac{1}{2}\left(-x+x^{2}\right), \quad B_{3}(x)=\frac{1}{6}\left(x-x^{3}\right) \\
& B_{4}(x)=\frac{1}{1680}\left(111 x-175 x^{3}+70 x^{4}-7 x^{6}+x^{7}\right), \\
& B_{5}(x)=\frac{1}{1680}\left(99 x-105 x^{3}+7 x^{6}-x^{7}\right) \\
& B_{6}(x)=\frac{1}{1680}\left(27 x-35 x^{3}+14 x^{5}-7 x^{6}+x^{7}\right), \\
& B_{7}(x)=\frac{1}{10080}\left(-895 x+35 x^{3}-7 x^{6}+3 x^{7}\right)
\end{aligned}
$$

Further, a seventh $P(x)$ on $[1,2]$ can be written as 


$$
\begin{aligned}
Q(x)= & Q(2) B_{1}(2-x)+Q(1) B_{0}(2-x) \\
& +Q^{\prime \prime}(2) B_{2}(2-x)+Q^{\prime \prime \prime}(1) B_{3}(2-x) \\
& +Q^{(4)}(1) B_{5}(2-x)-Q^{(4)}(2) B_{4}(2-x) \\
& -Q^{(5)}(2) B_{6}(2-x)+Q^{(6)}(1) B_{7}(2-x)
\end{aligned}
$$

It is easy to verify that a seventh $P(x)$ can be expressed in the following form:

$$
\begin{aligned}
P(x)= & P(0) A_{o}(x)+P(1) A_{1}(x) \\
& +P^{\prime}(0) A_{2}(x)+P^{\prime}(1) A_{3}(x) \\
& +P^{\prime \prime}(0) A_{4}(x)+P^{\prime \prime \prime}(1) A_{5}(x) \\
& +P^{(5)}(0) A_{6}(x)+P^{(6)}(1) A_{7}(x)
\end{aligned}
$$

where

$$
\begin{aligned}
& A_{0}(x)=\frac{1}{6}\left(6-35 x^{3}+35 x^{4}-7 x^{6}+x^{7}\right), \\
& A_{1}(x)=\frac{1}{6}\left(35 x^{3}-35 x^{4}+7 x^{6}-x^{7}\right) \\
& A_{2}(x)=\frac{1}{48}\left(48 x-199 x^{3}+181 x^{4}-35 x^{6}+5 x^{7}\right), \\
& A_{3}(x)=\frac{1}{16}\left(-27 x^{3}+33 x^{4}-7 x^{6}+x^{7}\right) \\
& A_{4}(x)=\frac{1}{48}\left(24 x^{2}-59 x^{3}+41 x^{4}-7 x^{6}+x^{7}\right), \\
& A_{5}(x)=\frac{1}{288}\left(11 x^{3}-17 x^{4}+7 x^{6}-x^{7}\right) \\
& A_{6}(x)=\frac{1}{1920}\left(5 x^{3}-15 x^{4}+16 x^{5}-7 x^{6}+x^{7}\right), \\
& A_{7}(x)=\frac{1}{17280}\left(-7 x^{3}+14 x^{4}-11 x^{6}+5 x^{7}\right)
\end{aligned}
$$

and a seventh $Q(x)$ on $[1,2]$ can be expressed as

$$
\begin{aligned}
Q(x)= & Q(2) A_{0}(2-x)+Q(1) A_{1}(2-x) \\
& +Q^{\prime}(1) A_{3}(2-x)+Q^{\prime}(2) A_{2}(2-x) \\
& +Q^{\prime \prime}(2) A_{4}(2-x)+Q^{\prime \prime \prime}(1) A_{5}(2-x) \\
& +Q^{(5)}(2) A_{6}(2-x)+Q^{(6)}(1) A_{7}(2-x)
\end{aligned}
$$

Also the following relations are obtained:

$$
\begin{aligned}
& A_{0}(2-x)=A_{1}(x), A_{1}(2-x)=A_{0}(x) \\
& A_{2}(2-x)=-A_{3}(x), A_{3}(2-x)=-A_{2}(x) \\
& A_{4}(2-x)=A_{4}(x), A_{5}(2-x)=-A_{5}(x) \\
& A_{6}(2-x)=-A_{6}(x), A_{7}(2-x)=A_{7}(x)
\end{aligned}
$$

Using (4) and (6), we have

$$
\begin{aligned}
P^{\prime \prime}(1)= & 7 P(0)-7 P(1)+\frac{23}{8} P^{\prime}(0) \\
& +\frac{33}{8} P^{\prime}(1)+\frac{3}{8} P^{\prime \prime}(0)+\frac{5}{48} P^{\prime \prime \prime}(1) \\
& +\frac{1}{960} P^{(5)}(0)-\frac{1}{2880} P^{(6)}(1)
\end{aligned}
$$

$$
\begin{aligned}
P^{\prime \prime \prime}(0)= & -35 P(0)+35 P(1)-\frac{199}{8} P^{\prime}(0) \\
& -\frac{81}{8} P^{\prime}(1)-\frac{53}{8} P^{\prime \prime}(0)+\frac{11}{48} P^{\prime \prime \prime}(1) \\
& +\frac{1}{64} P^{(5)}(0)-\frac{7}{2880} P^{(6)}(1) \\
P^{(4)}(0)= & 140 P(0)-140 P(1)+\frac{181}{2} P^{\prime}(0) \\
& +\frac{99}{2} P^{\prime}(1)+\frac{41}{2} P^{\prime \prime}(0)-\frac{17}{12} P^{\prime \prime \prime}(1) \\
& -\frac{3}{16} P^{(5)}(0)+\frac{1}{720} P^{(6)}(1)
\end{aligned}
$$

$$
\begin{aligned}
P^{(4)}(1)= & -140 P(0)+140 P(1)-\frac{169}{2} P^{\prime}(0) \\
& -\frac{111}{2} P^{\prime}(1)-\frac{29}{2} P^{\prime \prime}(0)+\frac{53}{12} P^{\prime \prime \prime}(1) \\
& -\frac{1}{16} P^{(5)}(0)+\frac{13}{720} P^{(6)}(1) \\
P^{(5)}(1)=- & 420 P(0)+420 P(1)-\frac{525}{2} P^{\prime}(0) \\
& -\frac{315}{2} P^{\prime}(1)-\frac{105}{2} P^{\prime \prime}(0)+\frac{35}{2} P^{\prime \prime \prime}(1) \\
& -\frac{5}{16} P^{(5)}(0)+\frac{13}{48} P^{(6)}(1) \\
& -315 P^{\prime}(1)-105 P^{\prime \prime}(0)+\frac{35}{2} P^{\prime \prime \prime}(1) \\
& -\frac{21}{8} P^{(5)}(0)-\frac{11}{24} P^{(6)}(1)
\end{aligned}
$$

Similarly using (5) and (6), we have

$$
\begin{aligned}
Q^{\prime \prime}(1)= & 7 Q(2)-7 Q(1)+\frac{23}{8} Q^{\prime}(2) \\
& +\frac{33}{8} Q^{\prime}(1)+\frac{3}{8} Q^{\prime \prime}(2)+\frac{5}{48} Q^{\prime \prime \prime}(1) \\
& +\frac{1}{960} Q^{(5)}(2)-\frac{1}{2880} Q^{(6)}(1) \\
Q^{\prime \prime \prime}(2)= & 35 Q(2)-35 Q(1)-\frac{199}{8} Q^{\prime}(2) \\
& -\frac{81}{8} Q^{\prime}(1)+\frac{59}{8} Q^{\prime \prime}(2)+\frac{11}{48} P^{\prime \prime \prime}(1) \\
& +\frac{1}{64} Q^{(5)}(2)+\frac{7}{2880} Q^{(6)}(1) \\
Q^{(4)}(1)= & -140 Q(2)+140 Q(1)-\frac{169}{2} Q^{\prime}(2) \\
& -\frac{111}{2} Q^{\prime}(1)-\frac{29}{2} Q^{\prime \prime}(2)+\frac{59}{12} Q^{\prime \prime \prime}(1) \\
& -\frac{1}{16} Q^{(5)}(2)+\frac{23}{720} Q^{(6)}(1)
\end{aligned}
$$




$$
\begin{aligned}
Q^{(4)}(2)= & 140 Q(2)-140 Q(1)+\frac{181}{2} Q^{\prime}(2) \\
& +\frac{99}{2} Q^{\prime}(1)+\frac{41}{2} Q^{\prime \prime}(2)-\frac{17}{12} Q^{\prime \prime \prime}(1) \\
& -\frac{3}{16} Q^{(5)}(2)+\frac{13}{720} Q^{(6)}(1) \\
Q^{(5)}(1)= & 420 Q(2)-420 Q(1)+\frac{525}{2} Q^{\prime}(2) \\
& +\frac{315}{2} Q^{\prime}(1)+\frac{105}{2} Q^{\prime \prime}(2)-\frac{35}{4} Q^{\prime \prime \prime}(1) \\
& +\frac{5}{16} Q^{(5)}(2)-\frac{13}{720} Q^{(6)}(1) \\
& -840 Q(2)+840 Q(1)-525 Q^{\prime}(2) \\
& -315 Q^{\prime}(1)-105 Q^{\prime \prime}(2)+\frac{35}{2} Q^{\prime \prime \prime}(1) \\
& -\frac{21}{8} Q^{(5)}(2)-\frac{11}{24} Q^{(6)}(1)
\end{aligned}
$$

Theorem 1 :( Existence and Uniqueness)

For every odd integer $n$ and for every set of $\frac{5 n+9}{2}$ real numbers $f_{\circ}, f_{1}, \ldots, f_{n} ; f_{1}^{\prime \prime}, f_{3}^{\prime \prime}, \ldots f_{n}^{\prime \prime} ; f_{0}^{(3)}, f_{2}^{(3)}{ }_{2}, \ldots, f_{n-1}^{(3)}$; $f_{1}^{(4)}, f_{3}{ }^{(4)}, \ldots, f_{n}{ }^{(4)}$, there exists a unique $S(x) \in S_{n, 7}^{(6)}$ denotes the class of all splines of degree $\leq 7$ which belongs to $C^{6}[0,1]$ and $\mathrm{n}$ is the number of knots satisfies all condition in (1).

\section{Proof of theorem 1:}

For a given $S(x) \in S^{(6)}{ }_{n, 7} S(x) \in S_{n, 7}^{(6)}$ set $h=n^{-1}$, $M_{v}=S^{(4)}(v h+), \quad v=0,1, \ldots \ldots, n-1, \quad N v=S^{(4)}(v h-)$, $v=0,1, \ldots, n$. Since $S^{(6)}(x)$ is linear in each internal $(v h,(v+1) h)$, it is completely determined by the $(2 n)$ constants $\left\{M_{v}\right\}_{v=0}^{n-1}$ and $\left\{N_{v}\right\}_{v=1}^{n}$. Also if $S(x)$ satisfies the requirements of Theorem 1 that for $2 v h \leq x \leq(2 v+1) h$, $v=0,1,2, \ldots .,\left(\frac{n-1}{2}\right)$, it must have the following form:

$$
\begin{aligned}
S(x)= & f_{2 v} B_{0}\left(\frac{x-2 v h}{h}\right)+f_{2 v+1} B_{1}\left(\frac{x-2 v h}{h}\right) \\
& +h^{2} f_{2 v}^{\prime \prime} B_{2}\left(\frac{x-2 v h}{h}\right)-h^{3} f_{2 v}^{\prime \prime \prime} B_{3}\left(\frac{x-2 v h}{h}\right) \\
& +h^{4} M_{2 v} B_{4}\left(\frac{x-2 v h}{h}\right)+h^{4} N_{2 v+1} B_{5}\left(\frac{x-2 v h}{h}\right) \\
& -h^{5} f_{2 v+1}^{(5)} B_{6}\left(\frac{x-2 v h}{h}\right)+h^{6} f_{2 v}^{(6)} B_{7}\left(\frac{x-2 v h}{h}\right)
\end{aligned}
$$

and for $(2 v+1) h \leq x \leq(2 v+2) h, v=0,1, \ldots, \quad\left(\frac{x-\overline{2 v+1} h}{h}\right)$, $S(x)$ has the form:

$$
\begin{aligned}
S(x)= & f_{2+2 v} B_{0}\left(\frac{\overline{2 v+2} h-x}{h}\right)+f_{2 v+1} B_{1}\left(\frac{\overline{2 v+2} h-x}{h}\right) \\
& +h^{2} f_{2 v+2}^{\prime \prime} B_{2}\left(\frac{\overline{2 v+2} h-x}{h}\right)-h^{3} f_{2 v+1}^{\prime \prime \prime} B_{3}\left(\frac{\overline{2 v+2} h-x}{h}\right) \\
& +h^{4} N_{2 v+2} B_{4}\left(\frac{\overline{2 v+2} h-x}{h}\right)+h^{4} M_{2 v+1} B_{5}\left(\frac{\overline{2 v+2} h-x}{h}\right) \\
& -h^{5} f_{2 v+1}^{(5)} B_{6}\left(\frac{\overline{2 v+2} h-x}{h}\right)+h^{6} f_{2 v+2}^{(6)} B_{7}\left(\frac{\overline{2 v+2 h}-x}{h}\right)
\end{aligned}
$$

We shall show that it is possible to determine the (2n) parameters $\left\{M_{v}\right\}_{v=0}^{n-1}$ and $\left\{N_{v}\right\}_{v=1}^{n}$, such that the function $S(x)$ given by (1) and (9) will also satisfy (2) in Theorem 1 , and $S^{\prime \prime}(x), S^{\prime \prime \prime}(x), S^{(5)}(x)$ and $S^{(6)}(x)$ will be continuous on $[0,1] . S(x)$ is continuous because of the interpolating condition (1) in Theorem $1, S^{\prime}(x)$ and $S^{(4)}(x)$ are continuous on $[0,1]$ except at the points $(2 v h)$ and $\overline{2 v+1} h$, respectively, $v=0,1,2, \ldots ., \frac{n-1}{2}$.

From (10) we see that (1) in Theorem 1 is equivalent to:

$$
\begin{aligned}
& 37 M_{0}+33 N_{1} \\
= & \frac{1}{18 h^{4}}\left\{10080 f_{0}-10080 f_{1}^{\prime}\right. \\
& +10080 h f_{1}^{\prime}-5040 h^{2} f_{0}^{\prime \prime}-1680 h^{3} f_{1}^{\prime \prime \prime} \\
& \left.-162 h^{5} f_{1}^{(5)}-31 h^{6} f_{0}^{(6)}\right\} \\
& 169 M_{n-1}+181 N_{n} \\
= & \frac{h^{-4}}{6}\left\{10080 f_{n-1}-10080 f_{n}^{\prime}\right. \\
& -5040 h^{2} f_{n}^{\prime \prime}-30 h^{2} f_{n-1}^{\prime \prime}+3360 h^{3} f_{n-1}^{\prime \prime \prime} \\
& \left.+258 h^{5} f_{n}^{(5)}+53 h^{6} f_{n-1}^{(6)}\right\}
\end{aligned}
$$

and taking the second, third, fifth and sixth order derivatives respectively of (9) and (10), and also satisfies

$$
\begin{gathered}
S^{\prime \prime}(\overline{2 v+2} h-)=S^{\prime \prime}(\overline{2 v+2} h+), \\
S^{\prime \prime \prime}(\overline{2 v+2} h-)=S^{\prime \prime \prime}(\overline{2 v+2} h+), \\
S^{(5)}(\overline{2 v+2} h-)=S^{(5)}(\overline{2 v+2} h+)
\end{gathered}
$$

and $S^{(6)}(\overline{2 v+2} h-)=S^{(6)}(\overline{2 v+2} h+)$ are equivalent to:

$$
\begin{gathered}
N_{2 v+1}-M_{2 v}=\frac{h^{2}}{3}\left(f_{2 v+1}^{(6)}+\frac{3}{h} f_{2 v+1}^{(5)}+\frac{1}{2} f_{2 v}^{(6)}\right) \\
M_{2 v}-N_{2 v+1}+N_{2 v+2}-M_{2 v+1} \\
=\frac{h^{3}}{3}\left(\frac{3}{2 h} f_{2 v+1}^{(6)}-\frac{3}{h^{2}} f_{2 v+2}^{(5)}-\frac{3}{h^{2}} f_{2 v+1}^{(5)}-\frac{3}{2 h} f_{2 v}^{(6)}\right) \\
\frac{h^{3}}{560}\left(37 M_{2 v}+33 N_{2 v+1}\right)+\frac{h^{3}}{1680}\left(181 N_{2 v+2}+169 M_{2 v+1}\right) \\
=-\frac{1}{h} f_{2 v+2}+\frac{h}{2} f_{2 v+1}-\frac{1}{h} f_{2 v}-\frac{1}{h} f_{2 v+1} \\
+\frac{h^{2}}{2} f_{2 v}^{\prime \prime}+\frac{h^{2}}{2} f_{2 v+1}^{\prime \prime}+\frac{h^{2}}{6} f_{2 v+1}^{\prime \prime \prime}+\frac{h^{2}}{3} f_{2 v+2}^{\prime \prime \prime} \\
+\frac{43 h^{4}}{1680} f_{2 v+2}^{(5)}+\frac{9 h^{4}}{560} f_{2 v+1}^{(5)}-\frac{53 h^{5}}{10080} f_{2 v+1}^{(6)}+\frac{31 h^{5}}{10080} f_{2 v}^{(6)}
\end{gathered}
$$

Thus, the theorem will be established if we show that the system of linear equations (11)-(15) has a unique solution. This end will be achieved by showing that the homogeneous system corresponding to (11)-(15) has only zero solution.

The following is the homogeneous system of equations for $v=0,1,2, \ldots ., \frac{n-3}{2}$ 


$$
\begin{aligned}
& 37 M_{0}+33 N_{1}=0 \\
& 169 M_{n-1}+181 N_{n}=0 \\
& N_{2 v+1}-M_{2 v}=0 \\
& M_{2 v}-N_{2 v+1}+N_{2 v+2}-M_{2 v+1}=0 \\
& 111 M_{2 v}+99 N_{2 v+1}+181 N_{2 v+2}+169 M_{2 v+1}=0
\end{aligned}
$$

Putting the values $M_{n-1}=-\frac{181}{169} N_{n}$ and from (16), we have the following, for $v=0,1,2, \ldots ., \frac{n-3}{2}$

$$
\begin{gathered}
N_{n-1}-M_{n-2}=0 \\
111 M_{n-3}+99 N_{n-2}+181 N_{n-2}+169 M_{n-1}=0 \\
\rightarrow N_{n-1}=-\frac{3}{5} M_{n-2}
\end{gathered}
$$

Form (16) we have $M_{0}=-\frac{33}{37} N_{1}$ and also from (17), we obtain

$$
M_{n-3}=N_{n-2}=M_{n-2}=N_{n-1}=0
$$

By the same manner we get $M_{0}=M_{1}=\ldots=M_{n-1}=0$, and $N_{1}=N_{2}=N_{3}=\ldots=N_{n}=0$, see (Saxena and Joshi, (1980) and Faraidun (2010)), to solution of the homogeneous system for $n=4 p$ and $n=4 p+2$. This completes the proof of the Theorem 1.

\section{Convergence Analysis}

In this section, we apply the spline function interpolation for finding the optimal error bound.

Lemma 1: let $f \in C^{7}[0,1], \mathrm{n}$ any odd integer and $h=n^{-1}$, then for $S_{n}(x) \equiv S_{n}(f, x)$ of theorem 1 , we have

$$
\left\|S_{n}^{\prime}(\overline{2 v+1} h)-f^{\prime}(\overline{2 v+1} h)\right\| \leq \frac{25}{243} h^{6} \theta_{0} \omega_{7}(v h),\left|\theta_{0}\right|<1 .
$$

and

$$
\left\|S_{n}(2 v h)-f^{\prime}(2 v h)\right\| \leq \frac{730}{1863} h^{6} \omega_{7}(2 h), \text { where } v=0,1,2, \ldots, \frac{n-1}{2}
$$

Where

$\omega_{7}(v h)=\operatorname{Max}\left\{\left|f^{(7)}(x)-f^{(7)}(y)\right|:\lceil x-y\rceil \leq v h, \forall x, y \in[0,1]\right\}$.

Proof: Since $S_{n}(x) \equiv S_{n}(f, x)$ is seven degree in $2 v h \leq x \leq(2 v+1) h$, we obtain from (7)

$$
\begin{aligned}
h^{3} S_{n}^{\prime \prime \prime}(2 v h)= & -35 f_{2 v}+35 f_{2 v+1}- \\
& \frac{199}{8} h S^{\prime}(2 v h)-\frac{81}{8} h S^{\prime}(\overline{2 v+1} h) \\
& -\frac{53}{8} h^{2} f_{2 v}^{\prime \prime}+\frac{11}{48} h^{3} f_{2 v+1}^{\prime \prime \prime} \\
& +\frac{h^{5}}{64} f_{2 v}^{(5)}-\frac{7}{2880} h^{6} f_{2 v+1}^{(6)}
\end{aligned}
$$

Similarly from (7), since $S(x)$ is seven degree in $(2 v+1) h \leq x \leq(2 v+2) h$, we have

$$
\begin{aligned}
h^{3} S_{n}^{\prime \prime \prime}(\overline{2 v+2} h)= & 35 f_{2 v+2}-35 f_{2 v+1} \\
& -\frac{199}{8} h S^{\prime}(\overline{2 v+2} h)-\frac{81}{8} h S^{\prime}(\overline{2 v+1} h) \\
& +\frac{53}{8} h^{2} f_{2 v+2}^{\prime \prime}+\frac{11}{48} h^{3} f_{2 v+1}^{\prime \prime \prime} \\
& +\frac{h^{5}}{64} f_{2 v+2}^{(5)}+\frac{7}{2880} h^{6} f_{2 v+1}^{(6)}
\end{aligned}
$$

Writing $(\mathrm{v}+1)$ for $(\mathrm{v})$ in $(20)$, when $v=0,1,2, \ldots .,\left(\frac{n-3}{2}\right)$, then subtracting with equation (21), we obtain

$$
\begin{aligned}
& \frac{81}{8} h S_{n}^{\prime}((2 v+1) h)-f_{2 v+1}^{\prime}-\left(S_{n}^{\prime}((2 v+3) h)-f_{2 v+3}^{\prime}\right] \\
= & 70 f_{2 v+2}-35 f_{2 v+1}+\frac{59}{4} h^{2} f_{2 v+2}^{\prime \prime} \\
& +\frac{11}{48} h^{3} f_{2 v+1}^{\prime \prime \prime}-\frac{11}{48} h^{3} f_{2 v+3}^{\prime \prime \prime}+\frac{7}{2880} h^{6} f_{2 v+1}^{(6)} \\
& +\frac{7}{2880} h^{6} f_{2 v+3}^{(6)}+\frac{81}{8} h f_{2 v+1}^{\prime}+\frac{81}{8} h^{3} f_{2 v+3}^{\prime \prime \prime}
\end{aligned}
$$

Setting,

$$
A_{v}=S_{n}^{\prime}(v h)-f^{\prime}(v h) \text { for } v=0,1,2, \ldots, n
$$

From equation (22)

$$
\begin{aligned}
& \frac{81}{8} h\left[A_{2 v+1}-A_{2 v+3}\right] \\
= & 70 f_{2 v+2}-35 f_{2 v+1}+\frac{59}{4} h^{2} f_{2 v+2}^{\prime \prime} \\
& +\frac{11}{48} h^{3} f_{2 v+1}^{\prime \prime \prime}-\frac{11}{48} h^{3} f_{2 v+3}^{\prime \prime \prime}+\frac{7}{2880} h^{6} f_{2 v+1}^{(6)} \\
& +\frac{7}{2880} h^{6} f_{2 v+3}^{(6)}+\frac{81}{8} h f_{2 v+1}^{\prime}+\frac{81}{8} h^{3} f_{2 v+3}^{\prime \prime \prime}
\end{aligned}
$$

Using Taylor series expansion on the right hand sides of the above equation, we get:

$$
\begin{aligned}
\frac{81}{8} h\left[A_{2 v+1}-A_{2 v+3}\right]=\frac{h^{7}}{1440}[ & 20 f_{\alpha v}^{(7)}-1280 f_{\beta v}^{(7)}+177 f_{\gamma v}^{(7)} \\
& \left.+7 f_{\theta v}^{(7)}+1296 f_{\phi v}^{(7)}-220 f_{\lambda v}^{(7)}\right]
\end{aligned}
$$

Fix $k, 0 \leq k \leq \frac{n-3}{2}$. On summing both sides of (24) for $\mathrm{v}=\mathrm{k}, \mathrm{k}+1, \ldots, \frac{n-1}{2}$ and using the fact that $\mathrm{A}_{\mathrm{n}}=0$, we have

$$
\begin{aligned}
A_{2 k+1}= & \frac{h^{3}}{3} \sum_{v=k}^{(n-3) / 2}\left[20 f_{\alpha v}^{(7)}-1280 f_{\beta v}^{(7)}+177 f_{\gamma v}^{(7)}\right. \\
& \left.+7 f_{\theta v}^{(7)}+1296 f_{\phi v}^{(7)}-220 f_{\lambda v}^{(7)}\right] \\
= & \frac{25}{243} h^{6} \theta_{0} \omega_{7}(v h),\left|\theta_{0}\right|<1 .
\end{aligned}
$$

This completes the proof of part 1 of lemma 1 . To proof of second part lemma1, since $\mathrm{S}(\mathrm{x})$ is seventh degree in $2 v h \leq x \leq \overline{2 v+1} h$ from (8), we have 


$$
\begin{aligned}
h^{2} S_{n}^{\prime \prime}(\overline{2 v+1} h)= & 7 f_{2 v}-7 f_{2 v+1}+\frac{123}{8} h S^{\prime}(\overline{2 v+1} h) \\
& +\frac{23}{8} h S^{\prime}(\overline{2 v+1} h)+\frac{3}{8} h^{2} f_{2 v}^{\prime \prime}+\frac{5}{48} h^{3} f_{2 v+1}^{\prime \prime} \\
& +\frac{h^{5}}{960} f_{2 v}^{(5)}-\frac{1}{2880} h^{6} f_{2 v+1}^{(6)}, v=0,1, \ldots ., \frac{(n-1)}{2} .
\end{aligned}
$$

Similarly, since $\mathrm{S}(\mathrm{x})$ is seventh degree in $(2 v+1) h \leq x \leq \overline{2 v+2} h$ from (8) for $v=0,1, \ldots ., \frac{(n-3)}{2}$, we have

$$
\begin{aligned}
h^{2} S_{n}^{\prime \prime}(\overline{2 v+1} h)= & 7 f_{2 v}-7 f_{2 v+1} \\
& -\frac{33}{8} h S^{\prime}(\overline{2 v+1} h)-\frac{23}{8} h S^{\prime}(\overline{2 v+1} h) \\
& +\frac{3}{8} h^{2} f_{2 v+2}^{\prime \prime}-\frac{5}{48} h^{3} f_{2 v+1}^{\prime \prime \prime} \\
& +\frac{h^{5}}{960} f_{2 v+2}^{(5)}-\frac{1}{2880} h^{6} f_{2 v+1}^{(6)}
\end{aligned}
$$

From the above two relations for $v=0,1, \ldots ., \frac{(n-3)}{2}$, we have

$$
\begin{aligned}
& \frac{23}{8} h\left[S_{n}^{\prime}((2 v) h)+S_{n}^{\prime}((2 v+1) h)\right] \\
= & 7 f_{2 v}-7 f_{2 v+1} \\
& -\frac{33}{4} h S^{\prime}(\overline{2 v+1} h)+\frac{3}{8} h^{2}\left(f_{2 v+2}^{\prime \prime}-f_{2 v}^{\prime \prime}\right) \\
& -\frac{5}{24} h^{3} f_{2 v+1}^{\prime \prime \prime}-\frac{h^{5}}{960}\left(f_{2 v+2}^{(5)}+f_{2 v}^{(5)}\right)
\end{aligned}
$$

For $\mathrm{v}=0$, we have an account of $A_{0}=0$, Using Taylor Series, we obtain

$$
\begin{aligned}
\frac{23}{8} h A_{2}= & f_{2}-7 f_{0} \\
& -\frac{33}{4} h f_{1}^{\prime}+\frac{3}{8} h^{2}\left(f_{2}^{\prime \prime}-f_{0}^{\prime \prime}\right) \\
& -\frac{5}{24} h^{3} f_{1}^{\prime \prime \prime}+\frac{h^{5}}{960}\left(f_{2}^{(5)}+f_{0}^{(5)}\right) \\
& -\frac{23}{8} h f_{0}^{\prime}-\frac{23}{8} h f_{2}^{\prime} \\
= & \frac{8}{45} h^{7} f_{\alpha_{1}}^{(7)}-\frac{33}{2880} h^{7} f_{\alpha_{2}}^{(7)} \\
& +\frac{1}{10} h^{7} f_{\alpha_{3}}^{(7)}-\frac{5}{576} h^{7} f_{\alpha_{4}}^{(7)} \\
& -\frac{1}{480} h^{7} f_{\alpha_{5}}^{(7)}-\frac{23}{90} h^{7} f_{\alpha_{6}}^{(7)}-\frac{23}{8} h A_{1} \\
= & \frac{h^{7}}{2880}\left[512 f_{\alpha_{1}}^{(7)}-33 f_{\alpha_{2}}^{(7)}\right. \\
& +288 f_{\alpha_{3}}^{(7)}-25 f_{\alpha_{4}}^{(7)} \\
& \left.-6 f_{\alpha_{5}}^{(7)}-736 f_{\alpha_{6}}^{(7)}\right]-\frac{33}{4} h A_{1}
\end{aligned}
$$

Therefore, $\frac{23}{8} h\left|A_{2}\right| \leq \frac{5}{18} h^{7} \theta_{1} \omega_{7}(2 h)+\frac{33}{4} h\left|A_{1}\right|$, where $\left|\theta_{1}\right|<1$. Using (18), we have

$$
\left|A_{2}\right| \leq \frac{730}{1863} h^{6} \omega_{7}(2 h)
$$

Lemma 2: let $A_{v}=S_{n}^{\prime}(v h)-f^{\prime}(v h)$ for $v=0,1,2, \ldots, n$, then

$$
\left|A_{2 v}-A_{2 v+4}\right| \leq \frac{4393}{415} h^{6} \omega_{7}(4 h)+\frac{8}{115} h^{6}\left\|f_{\alpha}^{(7)}\right\|
$$

Where $\left\|f^{(7)}\right\|=\operatorname{Max}\left\{\left|f^{(7)}(x)\right|: 0 \leq x \leq 1\right\}$

Proof: Setting $(v+1)$ for $\mathrm{v}$ in (27), we have for $v=0,1, \ldots ., \frac{n-3}{2}$.

$$
\begin{aligned}
& \frac{23}{8} h\left[S_{n}^{\prime}((2 v+2) h)+S_{n}^{\prime}((2 v+4) h)\right] \\
= & 7 f_{2 v+4}-7 f_{2 v+2} \\
& -\frac{33}{4} h S^{\prime}(\overline{2 v+3} h)+\frac{3}{8} h^{2}\left(f_{2 v+4}^{\prime \prime}-f_{2 v+2}^{\prime \prime}\right) \\
& -\frac{5}{24} h^{3} f_{2 v+3}^{\prime \prime \prime}-\frac{h^{5}}{960}\left(f_{2 v+4}^{(5)}+f_{2 v+2}^{(5)}\right)
\end{aligned}
$$

Subtracting (27) from (29), and using (23), we have $v=0,1, \ldots ., \frac{n-5}{2}$

$$
\begin{aligned}
\frac{23}{8} h\left[A_{2 v}-A_{2 v+4}\right]= & 7\left[f_{2 v+2}-f_{2 v}-f_{2 v+4}\right] \\
& -\frac{22}{27}\left[70 f_{2 v+2}-35 f_{2 v+1}-35 f_{2 v+3}\right. \\
& +\frac{59}{3} h^{2} f_{2 v+2}^{\prime \prime}+\frac{11}{48} h^{3} f_{2 v+1}^{\prime \prime \prime}-\frac{11}{48} h^{3} f_{2 v+3}^{\prime \prime \prime} \\
& \left.+\frac{7}{2880} h^{6} f_{2 v+1}^{(6)}+\frac{7}{2880} h^{6} f_{2 v+3}^{(6)}\right] \\
& -\frac{3}{8} h^{2}\left(f_{2 v+4}^{\prime \prime}-f_{2 v}^{\prime \prime}\right)+\frac{23}{8} h h^{2}\left(f_{2 v+4}^{\prime}-f_{2 v}^{\prime}\right) \\
& -\frac{5}{24} h^{3}\left(f_{2 v+1}^{\prime \prime \prime}-f_{2 v+3}^{\prime \prime}\right)+\frac{h^{5}}{960}\left(f_{2 v+4}^{(5)}-f_{2 v}^{(5)}\right)
\end{aligned}
$$

Using Taylor expansion, and after some calculations, we obtain

$$
\begin{aligned}
& \frac{23}{8} h\left[A_{2 v}-A_{2 v+4}\right] \\
= & \frac{11}{315} h^{7} f_{\theta_{1 v}}^{(7)}-\frac{1024}{45} h^{7} f_{\theta_{2 v}}^{(7)} \\
& -\frac{2464}{1701} h^{7} f_{\theta_{1 v}}^{(7)}+\frac{11}{1944} h^{7} f_{\theta_{3 v}}^{(7)} \\
& +\frac{99}{8} h^{7} f_{\theta_{4 v}}^{(7)}-\frac{1298}{405} h^{7} f_{\theta_{5 v}}^{(7)} \\
& -\frac{121}{15552} h^{7} f_{\theta_{6 v}}^{(7)}+\frac{121}{192} h^{7} f_{\theta_{7} v}^{(7)} \\
& -\frac{77}{38880} h^{7} f_{\theta_{8 v}}^{(7)}-\frac{77}{12960} h^{7} f_{\theta_{9 v}}^{(7)} \\
& -\frac{16}{5} h^{7} f_{\theta_{10 v}}^{(7)}+\frac{736}{45} h^{7} f_{\theta_{11 v}}^{(7)} \\
& -\frac{5}{576} h^{7} f_{\theta_{12 v}}^{(7)}+\frac{135}{192} h^{7} f_{\theta_{13 v}}^{(7)}+\frac{1}{120} h^{7} f_{\theta_{14 v}}^{(7)}
\end{aligned}
$$




$$
\begin{gathered}
\rightarrow \frac{23}{8} h\left[A_{2 v}-A_{2 v+4}\right]=\frac{16565528}{544320} h^{7} \omega_{7}(4 h)-\frac{108864}{544320} h^{7} f_{\alpha}^{(7)} \\
\rightarrow\left|A_{2 v}-A_{2 v+4}\right| \leq \frac{4393}{415} h^{6} \omega_{7}(4 h)+\frac{8}{115} h^{6}\left\|f_{\alpha}^{(7)}\right\|
\end{gathered}
$$

Theorem 2: Let $f \in C^{7}[0,1]$ and $\mathrm{n}$ an odd integer, then the unique seventh spline $S(x)$ satisfying conditions of Theorem1, we have

$$
\left\|S^{(r)}(x)-f^{(r)}(x)\right\|_{\infty} \leq \begin{cases}2 h^{6-r} K_{1} & \text { where } r=5,6 \\ h^{4-r}\left(2 K_{1} h^{2}+K_{2}\right) & \text { where } r=0,1,2,3,4 .\end{cases}
$$

Where

$$
K_{1}=\frac{675}{720} h \omega_{7}(f)+\frac{405}{720} h\left\|f^{(7)}\right\|+175 h^{-5}\left|A_{2 v}\right|+105 h^{-5}\left|A_{2 v+1}\right|,
$$

and $\omega_{7}($.$) denotes the modulus of continuity of f^{(7)}$.

Proof: Let $2 v h \leq x \leq \overline{2 v+1}, v=0,1, \ldots ., \frac{n-1}{2}$ from (9), we have

$$
\begin{aligned}
S^{(6)}(x) & =S^{(6)}(2 v h) B_{\circ}\left(\frac{x-2 v h}{h}\right)+S^{(6)}((2 v+1) h) B_{1}\left(\frac{x-2 v h}{h}\right) \\
& =S^{(6)}(2 v h) B_{1}\left(\frac{2 v+1 h-x}{h}\right)+S^{(6)}((2 v+1) h) B_{1}\left(\frac{x-2 v h}{h}\right),
\end{aligned}
$$

where $B_{1}\left(\frac{\overline{2 v+1} h-x}{h}\right)=B_{1}\left(\frac{x-2 v h}{h}\right)$

$$
\begin{aligned}
S^{(6)}(x)-f^{(6)}(x)= & \left(S^{(6)}(2 v h)-f^{(6)}(x)\right) B_{1}\left(\frac{x-2 v h}{h}\right) \\
& +\left(S^{(6)}((2 v+1) h)-f^{(6)}(x)\right) B_{1}\left(\frac{x-2 v h}{h}\right)
\end{aligned}
$$

Since $B_{1}\left(\frac{\overline{2 v+1} h-x}{h}\right)+B_{1}\left(\frac{x-2 v h}{h}\right)=1$ and $\left|B_{\circ}(x)\right| \leq 1$, $\forall x \in[0,1]$

$$
\left|S^{(6)}(x)-f^{(6)}(x)\right| \leq\left|S^{(6)}(2 v h)-f^{(6)}(x)\right|+\left|S^{(6)}(\overline{2 v+1} h)-f^{(6)}(x)\right| \leq 2 K_{1}
$$

Where

$$
K_{1}=\frac{675}{720} h \omega_{7}(f)+\frac{405}{720} h\left\|f^{(7)}\right\|+175 h^{-5}\left|A_{2 v}\right|+105 h^{-5}\left|A_{2 v+1}\right|
$$

\section{Since}

$$
S^{(5)}(x)-f^{(5)}(x)=\int_{x_{2 v}}^{x}\left(S^{(6)}(t)-f^{(6)}(t)\right) d t+S^{(5)}(2 v h)-f_{2 v}^{(5)}
$$

and $S^{(5)}(2 v h)=f^{(5)}(2 v h),\left|S^{(5)}(x)-f^{(5)}(x)\right| \leq 2 h K_{1}$, similarly $\left|S^{(4)}(x)-f^{(4)}(x)\right| \leq 2 h^{2} K_{1}+K_{2} \quad$, where $K_{2}=\left|S^{(4)}(2 v h)-f^{(4)}(2 v h)\right|$
$S^{(3)}(x)-f^{(3)}(x)=\int_{x_{2 v+1}}^{x}\left(S^{(4)}(t)-f^{(4)}(t)\right) d t+S^{(3)}(\overline{2 v+1} h)-f_{2 v+1}^{(3)}$

since $S^{(3)}(\overline{2 v+1} h)=f^{(3)}(\overline{2 v+1} h)$

$$
\rightarrow\left|S^{(3)}(x)-f^{(3)}(x)\right| \leq h\left(2 K_{1} h^{2}+K_{1}\right)
$$

similarly $\left|S^{\prime \prime}(x)-f^{\prime \prime}(x)\right| \leq h^{2}\left(2 K_{1} h^{2}+K_{2}\right)$

Since $S(2 v h)=f(2 v h) \quad, \quad S(\overline{2 v+1} h)=f(\overline{2 v+1} h)$, therefore $\quad S^{\prime}(\lambda)=f^{\prime}(\lambda) \quad, \quad 2 v h \leq \lambda \leq \overline{2 v+1} \quad$ and $\left|S^{\prime}(x)-f^{\prime}(x)\right| \leq h\left|S^{\prime \prime}(x)-f^{\prime \prime}(x)\right| \leq h^{3}\left(2 K_{1} h^{2}+K_{2}\right)$, similarly $|S(x)-f(x)| \leq h^{4}\left(2 K_{1} h^{2}+K_{2}\right)$.

\section{Conclusion}

In this paper, we apply the two inhomogeneous seventh spline interpolations for finding the best optimal errors bound, also order of spline and the boundary conditions are developed. Convergence analysis and basic properties of the inhomogeneous spline model has been proposed. Also, the continuity of derivatives across mesh points improves convergence for the spline function.

\section{References}

[1] Ahlberg, J. H.; Nilson, E. N. and Walsh, J. L., The theory of splines and their applications, New York, London, 1967.

[2] Athanassios Nikolis, Numerical solutions of ordinary differential equations with quadratic trigonometric splines, Applied Mathematics E-Notes, ISSN 1607-2510, Vol.4,(2004),p. 142-149.

[3] De Boor C., A Practical Guide to Splines. Revised Edn., Springer Verlag, New York, (2001).

[4] Faraidun K. Hama-Salh, Inhomogeneous Lacunary Interpolation by Splines $(0,2 ; 0,1,4)$ Case, Asian Journal of Mathematics and Statistics 3(4), (2010), p. 211-224.

[5] Howell, G. and Varma, A.K., Best Error Bounds for Quantic Spline Interpolation, Approximation Theory, Vol.58, No.1 (1989).

[6] Karwan H.F. Jwamer, Minimizing error bounds in $(0,2,3)$ lacunary interpolation by sextic spline function, Journal of Mathematics and Statistics ,USA, 3(4),(2007), pp. 249-256 .

[7] Karwan H.F. Jwamer and Saeed R.K, (0, 1, 3) Lacunary Interpolation with Splines of Degree Six, Journal of Applied and Industrial Sciences, April, 2013, 1 (1), p. 21-24.

[8] Meir, A. and A. Sharma, Lacunary interpolation by splines. SIAM J. Numer. Anal., 10,(1973),p. 433-442.

[9] Saad A Manna, Faraidun K. Hama-Salh and Hardi N. Aziz, An approximate solution of some differential equations with new type of interpolation, A. J. of M. and St., Vol. 6(1),(2013), p. 33-42.

[10] Saeed R.K. and Karwan H.F. Jwamer., Inhomogeneous Lacunary Interpolation, Journal of Dohuk University, Vol.6, No.1,(2003), p 94-104.

[11] Saxena, R.B. and T. C. Joshi,Inhomogeneous lacunary interpolation by splines, I $(0,2: 0,3)$. Bulgaricae Mathematicae Publi., vol. 6, (1980), p. 341-351. 